\title{
The Significance of a Neglected Romanian Book - "The Methods of the Clinical Laboratory", by Ioan Manta and Alexandru Ciplea
}

The book "The Methods of the Clinical Laboratory" - written by Ioan Manta (1900-1979) and Alexandru Ciplea (19121988) - was one of the most significant Romanian medical books of the first half of the $20^{\text {th }}$ century (Figure 1). It was edited in 1944, during the Second World War. It may be considered a premiere in Romanian medical literature and held an essential place in Romanian medical laboratories for more than a quarter of a century. Unfortunately, nowadays, 70 years since it was published for the first time, it has been almost forgotten by the majority of Romanian physicians.

It is obvious that in the 1950s and 1960s (when this book was greatly used), medical laboratory activities were more laborious compared with those in the present day and, consequently, required longer to obtain results. At that time, there were neither very rapid techniques, nor already prepared reagents. Even so, some techniques described in this book - for instance some microscopic investigations for parasitology and bacteriology - are still applicable today.

During the Second World War, few textbooks from abroad were used in Romania, and those that were available contained a panel with many laboratory techniques. Therefore, it was important to have a single book that used standard terms.

Both of the authors of this book were members of the Cluj Faculty of Medicine; Manta was a Professor in the Department of Biological Chemistry and Ciplea was a lecturer in the Second Clinic of Internal Medicine.

The authors were not interested in writing an original work, but wanted to present the medical techniques which, at that time, were considered standard. They offered laboratory physicians the most useful laboratory methods, which were simpler, more accurate and verified in the clinics and laboratories of the Cluj Faculty of Medicine. The methods were chosen according to the viewpoints of important foreign specialists in the domain of laboratory medicine. Sometimes, the authors presented different variants of the same technique, in order to offer physicians the most convenient method for different clinical laboratories. They mentioned the name of the author who developed each method, with some original techniques were initially described by Manta and Ciplea et al. (1).
The Rector of Cluj University, Professor Iuliu Haţieganu (1885-1959), wrote in the preface of this volume "the Romanian medical literature was enriched by this treatise and the authors contributed in a decisive manner to the development of the Romanian medical science prestige, giving to physicians and students a wonderful medical guide" (2).

The book contains over 800 laboratory techniques, including laboratory sampling and preservation techniques, different types of tests and methods for qualitative and quantitative determinations, techniques to prepare various solutions, stains, reagents and microbiological culture media, as well as techniques for the use of some laboratory equipment.

The book includes six chapters that deal with the following biological products: blood, cerebrospinal fluid, urine, gastric and duodenal juices, feces and sputum.

For each method, the authors presented the principle of the action, the reagents required, the working technique, and calculations, results and their interpretation.

At the beginning of each chapter, the following are presented: methods for the collection of fresh specimens, different ways of conservation, staining of smears for blood, sputum and fecal analysis, methods for obtaining cerebrospinal fluid, and how to perform sedimentation (from cerebrospinal fluid, urine, gastric juice). Also, details are given about parasitological and microbiological examinations used to detect the presence of parasites and pathogenic bacteria. To highlight the diversity of methods described in the book, it is sufficient to give two examples. In the chapter focused on blood, six techniques are described for the determination of clotting time, while there are eight qualitative methods to confirm albuminuria: the heating test, the Heller test, a method with potassium ferrocyanide, a method with metaphosphoric acid, a method with trichloroacetic acid, a method with sulfosalicylic acid, the Esbach method and the Tanret test.

Manta and Ciplea described the inaccuracies of some methods. For example, Manta recognized that in the Esbach test, in addition to albumin, the reagent also precipitates with mucoid substances, uric acid, alkaloids, etc. This problem was also reported by Pierre Thomas (1876-1963), who was Manta's forerunner at the Chair of Biological Chemistry in the Cluj Faculty of Medicine (3).

Address for Correspondence: Dr. Cristian Barsu, Faculty of Medicine, "Iuliu Hatieganu" University of Medicine and Pharmacy, Cluj-Napoca, Romania Phone: +40 0264599983 e-mail: cristianbarsu@yahoo.com

Received: 12.08.2015 Accepted: 22.10.2015 - DOI: 10.5152/balkanmedj.2015.1511452

Available at www.balkanmedicaljournal.org 


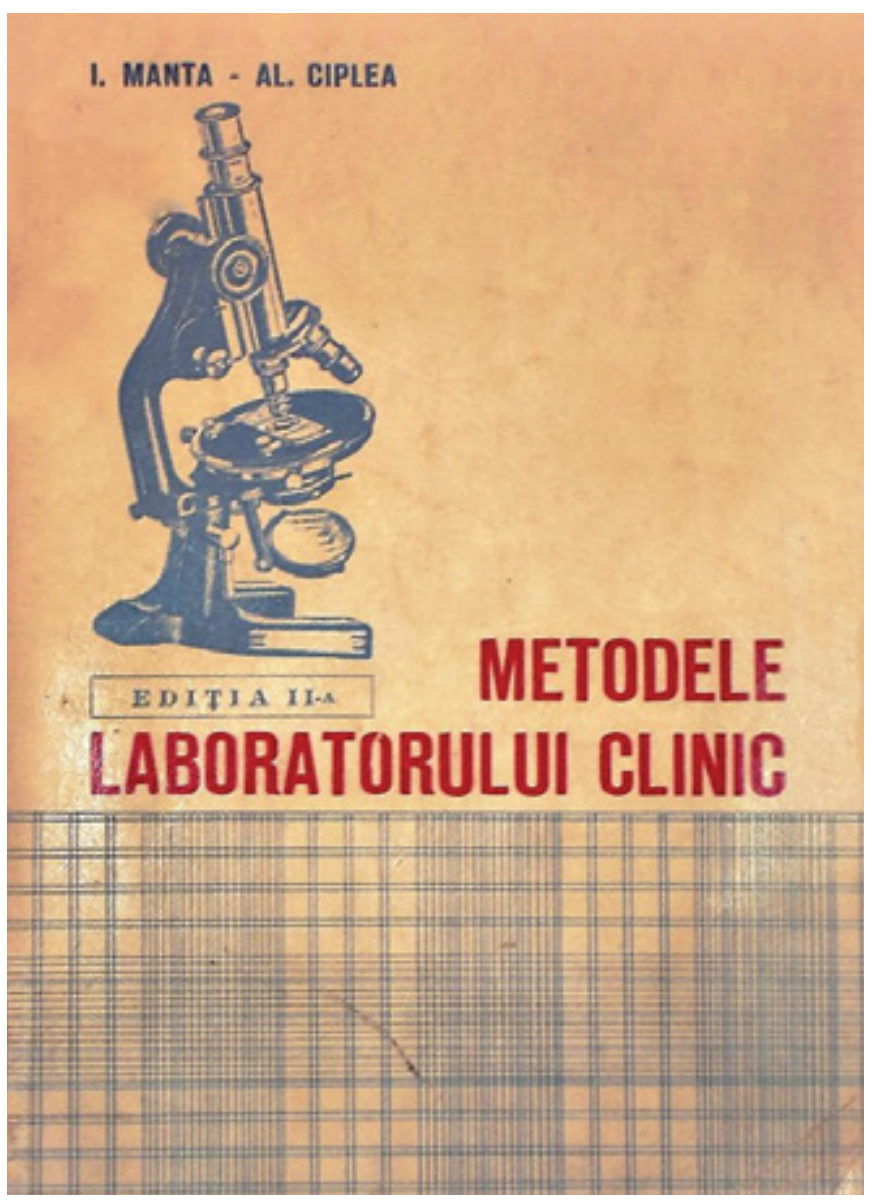

FIG. 1. The book's cover of the $2^{\text {nd }}$ edition

For blood, the methods for counting erythrocytes and leukocytes were presented in a very detailed manner. There was detail about three types of diluting pipettes: Potain, Thoma and Pappenheim, four types of counting chambers: Thoma, Türk, Bürker and Bürker-Türk, the determination of platelets using Kocher-Fonio stain and of reticulocytes with C.M. Laur and Fiessinger stains, as well as methods for counting, performing calculations and interpreting results. In the same chapter, Ciplea detailed methods to determine bleeding time, clotting time (applying six techniques, one of them being the historical "Schultz pearly capillary" method), the globular resistance of erythrocytes and sedimentation rates (using Westergren and Kowarski pipettes). They also mentioned the determinations of hemoglobin (utilizing Gowers-Sahli and Zeiss hemoglobinometers and a "sand clock"), hematocrit (with Hedin and Bönninger apparatus) and the alkaline reserve (with Van Slyke apparatus). However, the data for peripheral blood cells are presented in a short form. Methods for staining blood smears are included (Giemsa, May-Grünwald-Giemsa and Pappenheim stains), but without a leukocyte formula or descriptions of leukocyte cells such as lymphocytes, monocytes and granulocytes.
Related to the bacteriological blood laboratory methods, various methods for preparing bullions for hemocultures and their sterilization in autoclaves are mentioned. For example, five microbiological culture media and six Barsiekow media are presented, all of which can be used to isolate and identify typhoid, paratyphoid and coli bacilli. Also, some serological reactions are described, such as the agglutination test for typhoid fever (Widal reaction), the reaction for the diagnosis of brucellosis (Wright reaction) and the test for exanthematic fever diagnosis (Weil-Felix reaction). They also presented the complement fixation tests for echinococcosis (Weinberg-Pâr$\mathrm{vu}$ reaction), tests for the diagnosis of inflammatory disease (Bordet-Wassermann method and Bludau test) and flocculation reactions for the diagnosis of syphilis (Kahn, Meinicke and Sachs-Witebsky reactions).

A special chapter is focused on cerebrospinal fluid. This chapter describes the numbering of cells, using Fuchs-Rosenthal and Nageotte counting chambers, the chemical analysis of cerebrospinal fluid and different bacteriological methods, such as the determination of Neisseria meningitidis.

The chapter about urine begins with methods for urine collection, urine conservation, a description of the color and appearance of normal urine, and determination of the specific gravity using either an aerometer (urinometer) or pycnometer. Other methods presented in the same chapter are for the determination of ionic acidity using the Guillaumin method, total acidity with the titratable method of Folin, and interpretation of urinary $\mathrm{pH}$ according to different pathological statuses etc. It also includes some methods to measure the amount of mineral substances such as chloride (using Mohr and FreundTöpfer technique), phosphoric acid, sulfuric acid (RosenheimDrumond method) and urinary calcium. This chapter also contains the normal values for these substances.

To determine glucose, two qualitative methods are described: the Fehling and the Fischer tests. The absence of the qualitative Benedict test should be noted, although it was frequently used in many Romanian laboratories from the 1940s. Manta and Ciplea considered that the Fischer test also allowed a qualitative microscopic differentiation between three types of osazones: glucose, lactose and maltose, formed when sugars react with phenylhydrazine. In our opinion, it is less credible that the osazone differentiation was easy, although the drawings in this chapter show that glucose gives needle-shaped crystals, whereas maltose gives sunflower-shaped crystals.

Further on in the book, some quantitative determinations are detailed for glucose (Fehling and Bertrand methods), fructose (Seliwanoff reaction), lactose (Rubner-Buchner and Wöhlk methods) and galactose (Fissinger, Thiébaut and Dieryk technique) in urine. 
Regarding qualitative methods and tests for ketones, this book mentions the Legal method, the Libbrecht test and the Frommer-Emilewicz test for acetone. The Gerhardt test and Arreguine test were recommended for acetyl acetic acid. For the dosage of total acetone and beta-oxybutyric acid, the Van Slyke method and the Urbach colorimetric method were presented. Methods with the same principles for identifying different substances in urine as well as in the blood were only detailed in the chapter about blood.

Determinations of azotate substances in the urine included the total nitrogen dosage using the Parnas-Wagner apparatus (method which is described also for the dosage of total nitrogen in blood serum) and the ammonia dosage (the distillation Folin method and the permutite-Folin and Bell method, as well as the Urbach method).

Manta and Ciplea presented the Esbach method and the Bang gravimetric method as quantitative dosage techniques for albumin. Other methods are mentioned in order to confirm cystine and cysteine, arginine (Sakaguchi reaction), tyrosine (Millon reaction) and histidine (Kappeler-Adler method). For quantitative dosages of amino acids in the urine, the Sörensen method is mentioned. There are also some methods described for the dosage of uric acid, creatinine and indican (Jaffé, Obereyer, Jolles and Kumon methods). Qualitative methods for urobiline (Schlesinger method) and urobilinogen (Ehrlich-Bauer test), as well as the simultaneous dosage of urobiline and urobilinogen (Terwen, Heilmeyer and Krebs method) are also described.

To determine the origin of colored substances in the urine, the following methods are presented: the Heller test and oxidation tests with guaiac or benzidine to identify the presence of blood in the urine. There are also different methods presented, such as the Gmelin, Huppert-Salkowski and Hammarsten tests, to confirm the presence of bilirubin in the urine. Manta and Ciplea described determinations for bile acids using the Hay and Pettenkofer tests and qualitative tests for porphyrins. Manta also included the dosage of vitamins $\mathrm{B}_{1}$ and $\mathrm{C}$ in the urine. To identify sulfathiazole and sulfapyridine in the urine, which appear during treatment with sulfonamide drugs, the Oesterheld method is described. However, Manta and Ciplea omitted from this chapter the fact that other medicinal substances may be present in the urine, such as iodides, bromides, arsenic, salicylic acid, methylene blue, quinine etc. These methods were described in other books, for example in the textbook written by A. Desgrez in 1921 (4).

The bacteriological examination of urine is limited to the Tillmanns-Ohnesorge method for identifying some bacteria such as coli, lactis aerogenes, micrococcus ureae, Neisseria gonorrhoeae and Koch's bacillus.

The chapter on gastric and duodenal juice describes the chemical analysis of these two biological products. This chap- ter also includes bile examination. The description of bile is made according to the presentation by Ioan Goia (1892-1982) - Professor of Semeiology at the Cluj Faculty of Medicine (5).

A special chapter focused on coprology with presentation of the chemical analysis of fecal matter. The methods of bacteriology and examinations of sediments of fecal matter are also presented in detail.

In the chapter on sputum, the most important examinations are focused on the macroscopic and microscopic analyses of this biologic product. Bacteriological analyses are reduced to the Tillmanns-Ohnesorge method to identify Koch's bacillus, Friendlander's pneumobacillus, Haemophilus influenza, Bordet-Gengou bacillus etc.

At the end of this book, four short chapters are included, which are useful in completing the laboratory knowledge of a physician who is less familiar with different techniques for laboratory analyses.

One of the final chapters presents the physical methods used in clinical laboratory. It includes colorimetry, photometry, nephelometry, polarimetry and refractometry. There are also some general aspects noted and schemes for the Duboscq and Authenrieth-Königsberger colorimeters and the Pulfrich (Stufo) Zeiss and Leitz (Leifo) photometers etc.

Another short final chapter is dedicated to $\mathrm{pH}$ measurements. It contains some indicators in Sörensen, Clark and Lubs, Michaelis, Bogen and Kolthoff tables. There are also colorimetric techniques described for $\mathrm{pH}$ measurements, some of them using buffer solutions, others without and a photometric method with a Pulfrich (Zeiss) photometer. At the end of this chapter, blood $\mathrm{pH}$ determination is described using two colorimetric methods: Cullen and Hastings-Sendroy.

In the next short chapter, some preparation techniques for titrated solutions are revealed, along with tables of densities and concentrations of a number of acids and bases and also how to prepare different grades of alcohol.

The "Addendum" deals with OAB and MN blood systems, for blood group determination, with the Rh factor being presented only in the second edition of the book. An analysis of transudates, exudates, synovial and cystic fluids is also presented, which includes laboratory examinations in diphtheric angina, scarlet fever and meningococcal inflammation.

The book contains 35 references, written in Romanian, German and French.

The first edition of the book written by Manta and Ciplea was printed in elegant conditions, with 103 illustrations in the text, by the "Romanian Book" publishing house in Cluj. The second edition was printed three years later (in 1947), in much less technical conditions.

Regarding the first edition, it contains 543 pages, divided into ten chapters. 
The second edition has only 476 pages and eleven chapters. In this edition, the authors tried to eliminate errors that appeared in the first edition and omit those methods that were considered impracticable or unnecessary. They also added some new methods, which had proved to be useful for physicians. They improved the text by the inclusion of additional chapters, such as the chemical analysis and hygienic determinations of milk. The iconography was improved by adding a further 35 figures and the bibliography was extended by 9 additional titles.

Although for a quarter of century this book was very useful in almost all clinical laboratories in Romania and in many departments of biochemistry of different faculties from our country, it was considered a basic book, but, paradoxically, very little has been written since 1948 . The reason lies in the fact that both authors became political undesirables at the beginning of the communist period. Ioan Manta was thrown out of the educational system in 1949 and imprisoned between 1949 and 1951 (6). In 1952, he was allowed to continue his academic career in Cluj, starting in the Faculty of Pharmacy. In 1953, he came back as the Chair of Biological Chemistry from the Faculty of Medicine (7). Alexandru Ciplea was also eliminated from the academic educational system in 1952. In 1960, he became a researcher at the Physiology Department led by Prof. Grigore Benetato (1905-1972) at the Bucharest Institute of Medicine and Pharmacy.

In conclusion, there are sufficient arguments to consider this book as having a very important place in the Romanian history of laboratory medicine, because it marked a step in its evolution.

\section{Cristian Barsu}

Department of History of Medicine, Social Sciences and Humanism, "Iuliu Hatieganu" University of Medicine and Pharmacy, Cluj-Napoca, Romania

\section{REFERENCES}

1. Manta I. Introducere (Introduction). In: Manta I, Ciplea Al, editors. Metodele Laboratorului Clinic (The Methods of the Clinical Laboratory). Sibiu: Cartea Romaneasca din Cluj (Romanian Book from Cluj) Publ. House; 1944:V.

2. Hatieganu I. Prefata (Preface). In: Manta I, Ciplea Al, editors. Metodele Laboratorului Clinic (The Methods of the Clinical Laboratory). Sibiu: Cartea Romaneasca din Cluj (Romanian Book from Cluj) Publ. House; 1944:VI.

3. Thomas P. Manuel de Bichimie (Biochemistry Textbook). Paris: Masson et Cie Éditeurs; 1936:866.

4. Desgrez A. Précis de Chimie Médicale (Textbook of Medical Chemistry). Paris: Librairie J.-B. Baillière et fils; 1921:442-5.

5. Goia I. Ficatul. Notiuni anatomo-morfo-clinice The Liver. Anatomo-morpho-physiological notions. In: Hatieganu I, Goia I, Hanganut M, Sparchez T, editors. Tratat Elementar de Semiologie si Patologie Medicala (The Medical Semiology and Pathology Elementary Treatise), vol. III. Cluj: Cartea Romaneasca (Romanian Book) Publ. House; 1938.

6. Barsu C. Testimonials about the life of Prof. Ion Manta (19001979), a victim of communist regime. Cj Med 2013;86:388-9.

7. Mircioiu C. Alexandru Ciplea. In: Simiti I, editor. Figuri Reprezentative ale Medicinii si Farmaciei Clujene (Representative Figures of Cluj Medicine and Pharmacy), vol. IV. Cluj-Napoca: IMF Printing House 1988:17-24.

\section{Comment on The Significance of a Neglected Romanian Book - "The Methods of the Clinical Laboratory", by Ioan Manta and Alexandru Ciplea}

I am very pleased to comment such an article reviewing the book "Metodele laboratorului clinic" (title in English: "The Methods of the Clinical Laboratory") (1) since I know one of the authors very well, as well as the importance of the book.

Professor Ioan Manta was the Founder and for several decades the Chairman of the Department of Medical Biochemistry of the University of Medicine and Pharmacy in Cluj-Na- poca, Romania. As other students in medicine, I was greatly impressed by Professor Manta's outstanding lectures, in which the great importance of biochemistry and molecular biology for medicine (and for the clinical laboratory in particular) was emphasized. Some of his students decided to engage in research in his department during their medical studies. Professor Manta ensured an excellent environment for both teach- 